\title{
ORDERED SPACES ALL OF WHOSE CONTINUOUS IMAGES ARE NORMAL
}

\author{
WILLIAM FLEISSNER AND RONNIE LEVY
}

(Communicated by Dennis K. Burke)

\begin{abstract}
Some spaces, such as compact Hausdorff spaces, have the property that every regular continuous image is normal. In this paper, we look at such spaces. In particular, it is shown that if a normal space has finite Stone-Cech remainder, then every continuous image is normal. A consequence is that every continuous image of a Dedekind complete linearly ordered topological space of uncountable cofinality and coinitiality is normal. The normality of continuous images of other ordered spaces is also discussed.
\end{abstract}

Let $X$ be a normal topological space. In general, there is no reason to expect every regular continuous image of $X$ to be normal. After all, every space, normal or not, is the continuous image of a normal space, namely, a discrete space. On the other hand, every continuous image of certain spaces is normal. For example, if $X$ is compact Hausdorff, or, more generally, regular Lindelöf, then every (regular) continuous image of $X$ is normal, because every continuous image of $X$ is Lindelöf. In this note, we discuss spaces with the property that every regular continuous image is normal. In particular, we point out that those metric spaces which have this property are the separable ones, and, we discuss the situation for complete linearly ordered topological spaces.

We make the convention that all given spaces are assumed to be regular, which includes Hausdorff. All metric spaces will be assumed to have the metric topology. All linearly ordered spaces will be assumed to have the order topology. Ordinals will be von Neumann ordinals and cardinals will be initial ordinals.

\section{ADJUNCTION SPACES, CLOSED SUBSETS, AND METRIC SPACES}

The construction of adjunction spaces is covered in several texts, such as [D, pp. 127-128]. We review the basics here. Let $f: A \rightarrow Y$ be a continuous map from a closed subset $A$ of a space $X$ to $Y$, where we may assume without loss of generality that $X \cap Y=\varnothing$. Generate an equivalence relation on $X \cup Y$ by $a \simeq f(a)$ for all $a$ in $A$. The resulting quotient space is denoted $X \cup_{f} Y$. It is

Received by the editors August 27, 1987 and, in revised form, January 12, 1988.

1980 Mathematics Subject Classification (1985 Revision). Primary 54C05, 54D15.

The first author gratefully acknowledges the support of Grant DMS-8603227 from the National Science Foundation. 
trivial to prove that (i) $Y$ is homeomorphic to a closed subset of $X \cup_{f} Y$ and (ii) the projection $p: X \cup Y \rightarrow X \cup_{f} Y$ is a continuous surjection. The method of proof (but not the statement) of [D, $\S$ VII 3.4, p. 145], yields the following.

1.1. Proposition. (a) Let $X$ be normal, $A$ a closed subset of $X, f: A \rightarrow Y$ continuous, and $Y$ regular. Then $X \cup_{f} Y$ is regular.

(b) If $Y$ is the union of finitely many closed normal subspaces, then $Y$ is normal.

(c) If $Y=K \cup N$, where $K$ is compact and $N$ is normal, then $Y$ is normal.

1.2. Proposition. $A$ space $X$ has the property that every continuous regular image is normal if and only if every closed subset has the property that every closed regular image is normal.

Proof. Since $X$ is a closed subspace of itself and the identity map from $X$ to itself is onto, it will suffice to show that if $f: A \rightarrow Y$ is a continuous surjection, $A$ is closed in $Y$, and $Y$ is not normal, then there is a continuous map $g$ from $X$ onto a nonnormal space. For this, let $Z=X \cup_{f} Y$ and let $g$ be the quotient map.

1.3. Corollary. If $X$ is a metric space, then every continuous image of $X$ is normal if and only if $X$ is separable.

Proof. A separable metric space is Lindelöf. A nonseparable metric space contains an uncountable closed discrete subspace which maps continuously onto the Tychonov plank.

\section{NORMAL ALMOST COMPACT SPACES}

To smooth the exposition in later sections, we record in this section some useful elementary results. A completely regular space $X$ is said to be almost compact if $\beta X \backslash X$ has at most one point. (See [GJ, p. 95].) If $\beta X \backslash X$ has exactly one point, denote the point by $\infty_{X}$.

2.1. Lemma. (a) $A$ (regular) space $X$ is almost compact and normal if and only if for any pair of disjoint closed subsets of $X$, at least one is compact.

(b) Every almost compact space is locally compact.

(c) Every continuous image of a normal almost compact space is normal and almost compact.

(d) If $X$ is almost compact and a dense proper subset of a completely regular space $Y$, then $Y=\beta X$.

Proof. (a) Sufficiency is immediate from problem $6 \mathrm{~J}$ of [GJ]. For necessity notice that if $X$ is normal and almost compact but not compact, then of any pair of disjoint closed subsets of $X$, at most one can have the point $\infty_{X}$ in its closure, so at most one of the sets is not compact. The proof of (b) is immediate from the fact that an almost compact space has compact Stone-Čech remainder. The proof of (c) is easy and omitted. For (d), note that $\beta X$ maps onto $\beta Y$ and hence $\beta Y \backslash X$ has as at most one point. 
2.2. Lemma. Suppose that $f$ is a continuous function from a normal almost compact space $X$ onto a space $Y$. From 2.1(c) we may consider $\beta f: \beta X \rightarrow$ $\beta Y$.

(a) If $\beta f\left(\infty_{X}\right) \in Y$, then $Y$ is compact.

(b) If $\beta f\left(\infty_{X}\right) \notin Y$, then $f$ is a closed map.

(c) If for some compact $K \subset Y$, the set $f^{\leftarrow}(K)$ is not compact, then $Y$ is compact.

Proof. (a) In this case, $Y$ is the continuous image of the compact space $\beta \mathrm{X}$. (b) If $H$ is closed in $X$, then $f^{\rightarrow}(H)=\beta f^{\rightarrow}\left(\mathrm{Cl}_{\beta X} H\right) \cap Y$. (c) $\infty_{X} \in$ $\mathrm{Cl}_{\beta X}\left(f^{\leftarrow}(K)\right)$ so $\beta f\left(\infty_{X}\right) \in K$ and (a) applies.

2.3. Lemma. Let $Z$ be a normal almost compact subset of $W$. Then either $Z$ is closed in $W$ or $\mathrm{Cl}_{W} Z$ is compact. Hence, in either case, $\mathrm{Cl}_{W} Z$ is normal.

Proof. If $\mathrm{Cl}_{W} Z$ had two disjoint noncompact closed subsets, their traces on $Z$ would be disjoint noncompact closed subsets of $Z$. Therefore, $\mathrm{Cl}_{W} Z$ is almost compact and normal. If there is an element of $\mathrm{Cl}_{W} Z \backslash Z$, then by 2.1 (d) $\mathrm{Cl}_{W} Z=\beta Z$.

\section{COFINALITY AND COMPLETE ORDERED SPACES}

In this section, we discuss linearly ordered spaces with the property that every continuous image is normal. In particular, we discuss those complete ordered spaces for which the confinality determines whether or not every continuous image is normal. For example, we show that if $X$ is a linearly ordered topological space which has a countably infinite co-initial subset but cofinality $\omega_{1}$, then there is a nonnormal continuous image of $X$.

In the proof of the following lemma, when we talk about a Tychonov plank, we mean a space of the form $(\alpha+1) \times\left(\omega_{0}+1\right)-\left\{\left(\alpha, \omega_{0}\right)\right\}$, where $\alpha$ is a cardinal of uncountable cofinality. The corner point and the top and right edges are defined in analogy with the usual Tychonov plank.

3.1. Lemma. Suppose $\alpha$ is a cardinal of uncountable cofinality such that $\omega_{0}<$ $\alpha \leq$ c. Let $X$ be the discrete union of $\alpha$ and $\omega_{0}$. Then there exists a continuous function from $X$ onto a nonnormal space $Y$.

Proof. Since the space $\alpha+1$ has a base of at most c clopen sets, the compact space $(\alpha+1) \times\left(\omega_{0}+1\right)$ is embedded in the separable space ${ }^{\mathbf{c}} 2 \times\left(\omega_{0}+1\right)$. It follows that there exists a space $Z$ such that the Tychonov plank $P=(\alpha+1) \times$ $\left(\omega_{0}+1\right)-\left\{\left(\alpha, \omega_{0}\right)\right\}$ is a closed subset of $Z$ and $Z-P$ is a countable dense subset of $Z$. Let $Y$ be the subspace of $Z$ consisting of $Z-P$ with the top edge $T$ and the right edge $R$ of $P$. Then, since $Y$ is a union of a copy of $\alpha$ and a countable set, $Y$ is a continuous image of $X$. Furthermore, $Y$, being a subset of a compact Hausdorff space, is regular. What is left to show is that $Y$ is not normal. We will show that $R$ and $T$ cannot be separated in $Y$. Suppose $U$ and $V$ are open subsets of $Y$ containing $R$ and $T$ respectively. Then there 
exist open subsets $U^{\#}$ and $V^{\#}$ of $Z-\left\{\left(\alpha, \omega_{0}\right)\right\}$ whose traces on $Y$ are $U$ and $V$ respectively. Since the right and top edges of $P$ are not separated by open sets, $U^{\#} \cap V^{\#} \neq \varnothing$, and since $Z-P$ is dense in $Z-\left\{\left(\alpha, \omega_{0}\right)\right\}$, there exists an $x \in Z-P$ such that $x \in U^{\#} \cap V^{\#}$. But then $x \in U \cap V$, so $U$ and $V$ are not disjoint. Therefore, $Y$ is not normal.

3.2. Corollary. Suppose $X$ is a linearly ordered topological space and $\alpha$ is a cardinal of uncountable cofinality such that $\omega_{1} \leq \alpha \leq \mathbf{c}$. If $X$ has countably infinite co-initiality and cofinality $\alpha$, or if $X$ has co-initiality $\alpha$ and countably infinite cofinality, then $X$ admits a map onto a nonnormal (regular) space.

Proof. In this case either $X$ has a closed subset homeomorphic to the discrete union of $\omega_{0}$ and $\alpha$ or $X$ has an uncountable closed discrete subset. Now apply 1.2 and either 1.3 or 3.1 .

Suppose that $X$ is a linearly ordered topological space such that the order has a first but no last element. Then it is not necessarily the case that every continuous image of $X$ is normal. In fact, it is easy to see that every infinite discrete space can be written in this form. However, if the order on $X$ is Dedekind complete, then every continuous image of $X$ is normal. This fact follows from the next observations.

3.3. Proposition. Suppose $X$ is a normal space such that $\beta X-X$ is finite. Then every continuous image of $X$ is normal.

Proof. Suppose $f: X \rightarrow Y$ is a continuous surjection. Let $\mathscr{N}=\left\{N_{p}: p \in\right.$ $\beta X-X\}$ be a disjoint collection of closed subsets of $X$ such that $\{p\} \cup N_{p}$ is a neighborhood of $p$. Notice that $M=X \backslash \bigcup\left\{\operatorname{Int}\left(N_{p}\right): p \in \beta X \backslash X\right\}$ is compact and therefore normal and almost compact. Since $N_{p}$ is a closed subset of the normal space $X, \beta N_{p}=\mathrm{Cl}_{\beta X}\left(N_{p}\right)=N_{p} \cup\{p\}$. Hence $N_{p}$ is normal and almost compact. Set $\mathscr{N}^{\prime}=\mathscr{N} \cup\{M\}$. Now $Y=\bigcup\left\{\mathrm{Cl}_{Y} Z: Z \in \mathscr{N}^{\prime}\right\}$, where by 2.3 each $\mathrm{Cl}_{Y} Z$ is normal. Hence, by $1.1(\mathrm{~b}), Y$ is normal.

Let us say that if a linearly ordered topological space has a first (last) element, then its co-initiality (cofinality) is 1 .

3.4. Corollary. Let $X$ be a complete linearly ordered topological space. Every continuous image of $X$ is normal if any of the following cases holds.

(a) Co-initiality of $X \leq \omega$ and cofinality of $X \leq \omega$.

(b) Co-initiality of $X=1$ and cofinality of $X>\omega$.

(c) Co-initiality of $X>\omega$ and cofinality of $X>\omega$.

Proof. In case (a) $X$ is $\sigma$-compact. In case (b) $X$ is normal and almost compact so 2.1 (c) applies. In case (c), $|\beta X \backslash X|=2$ and 3.3 applies.

\section{OTHER COMPLETE ORDERED SPACES}

In looking at the question of which complete ordered spaces have the property that every continuous image is normal, we have not considered the case 
where the order has countable co-initiality and cofinality greater than c. In this section, we look at this question.

4.1. Proposition. If $\alpha$ is any uncountable regular cardinal, then there exists a complete ordered space $X$ such that $X$ has countable co-initiality, the cofinality of $X$ is $\alpha$, and some continuous image of $X$ is not normal.

Proof. For an ordered space $A$, let $A_{\text {rev }}$ denote the ordered space obtained by reversing the order on $A$. Let $S$ be the set $\omega \times(\alpha+1)$ with the lexicographic order. Let $X$ be the union of $S_{\text {rev }}$ and $\alpha$ ordered so that every element of $\alpha$ is larger than every element of $S_{\text {rev }}$. Then clearly $X$ is order complete, $X$ has co-initiality $\omega$ and cofinality $\alpha$. To find a nonnormal space $Y$ which is the continuous image of $X$, let $Y$ be $[(\alpha+1) \times(\omega+1)]-(\alpha, \omega)$. Then $Y$ is nonnormal, just as the Tychonov plank is nonnormal-in fact, if $\alpha=\omega_{1}$, then $Y$ is the Tychonov plank. A continuous function from $X$ onto $Y$ is given by mapping $\{n\} \times(\alpha+1)$ onto $(\alpha+1) \times\{n\}$ for $n \in \omega$ and mapping $\alpha$ onto $\alpha \times\{\omega\}$ in the obvious ways.

Although 4.1 shows that there is no result which asserts that ordered spaces with countable co-initiality and large cofinality have normal images, there is such a result if the ordered space is nice enough.

4.2. Proposition. Suppose $X$ is the discrete union of $\omega$ and $\alpha$ where $\alpha$ is an ordinal of cofinality greater than c. Then every continuous image of $X$ is normal.

Proof. Suppose $f: X \rightarrow Y$ is a continuous surjection. The plan is to apply 1.1(b) to $Y=\mathrm{Cl}_{Y} f \rightarrow(\omega) \cup \mathrm{Cl}_{Y} f^{\rightarrow}(\alpha)$. By $2.3 \mathrm{Cl}_{Y} f^{\rightarrow}(\alpha)$ is normal. We will show that $\mathrm{Cl}_{Y} f \rightarrow(\omega)$ is normal by showing that it is a closed subset of a $\sigma$-compact (hence normal) space. If $f^{\rightarrow}(\alpha)$ is compact, then $Y$ is itself $\sigma$-compact and we are done. Therefore, we may suppose that $f^{\rightarrow}(\alpha)$ is not compact. By 2.2(c), then, for each compact subset $K$ of $f^{\rightarrow}(\alpha)$, the set $(f)$ $\alpha)^{\leftarrow}(K)$ is bounded in $\alpha$.

Every separable space, in particular $\mathrm{Cl}_{Y}\left(f^{\rightarrow}(\omega)\right)$, has weight at most c. By 2.1(b), $f^{\rightarrow}(\alpha)$ is locally compact. Thus, $\mathrm{Cl}_{Y}\left(f^{\rightarrow}(\omega)\right) \cap f^{\rightarrow}(\alpha)$ can be covered by c compact sets; call them $K_{\lambda}$. By (ii), each of the sets $f^{\leftarrow}\left(K_{\lambda}\right)$ is bounded and since $\alpha$ has cofinality greater than $\mathbf{c}$, there is a $\delta<\alpha$ such that $\mathrm{Cl}_{Y}\left(f^{\rightarrow}(\omega)\right) \subset f^{\rightarrow}(\omega) \cup f^{\rightarrow}(\delta+1)$, which is $\sigma$-compact.

\section{BIBLIOGRAPHY}

[D] J. Dugundji, Topology, Allyn and Bacon, Boston, Mass., 1966.

[GJ] L. Gillman and M. Jerison, Rings of continuous functions, Springer-Verlag, New York and Berlin, 1960. 15260

Department of Mathematics, University of Pittsburgh, Pittsburgh, Pennsylvania

Department of Mathematics, George Mason University, Fairfax, Virginia 22030

Department of Mathematics, University of Kansas, Lawrence, Kansas 66045 (Current address of William Fleissher) 